\title{
Activity differences of individually and group-housed male and female rats
}

\author{
J. C. DALRYMPLE-ALFORD and D. BENTON \\ University College of Swansea, Singleton Park, Swansea SA2 8PP, Wales
}

\begin{abstract}
Previous comparisons of the activity differences of individually and group-housed rats have produced inconsistent findings; therefore, we examined activity using three measures: openfield ambulation, nonspecific circadian activity, and rearing behavior. Open-field ambulation in two 5-day tests confirmed our prediction that isolates would be initially less active but later more active than group-housed rats. Isolates habituated more slowly to the activity monitor and tended to be more active during the light half of the 24-h cycle. In the rearing test, isolates showed elevated and more persistent activity than group-housed rats. All these activity differences were prevalent in both male and female rats. The results are interpreted in terms of the influence of differential housing on "fear responses," exploration of a novel environment, and differences in habituation/adaptation processes.
\end{abstract}

Although many studies have investigated the activity differences of individually and group-housed rats, they have often produced conflicting results. For example, in open-field studies several workers have found individually housed rats to be significantly more active than group-housed rats (Domjan, Schorr, \& Best, 1977; Einon \& Morgan, 1977; Sahakian, Robbins, \& Iverson, 1977). The opposite result, greater open-field motor activity in group-housed than in isolated rats, has also been reported regularly (Ader \& Friedman, 1964; Moyer \& Korn, 1965; Stern, Winokur, Eisenstein, Taylor, \& Sly, 1960). It is important to clarify this issue because isolation-induced behavioral changes have often been suggested as animal models of abnormal behavior (Nishikawa \& Tanaka, 1978; Speiser \& Weinstock, 1973).

It seemed pertinent that reports indicating lower open-field ambulation in isolates have utilized naive animals and have typically tested them either for a brief single trial (Ader, 1965) or for a few trials of short duration (Taylor, 1969). In contrast, reports of open-field hyperactivity in individually housed rats have usually used handled, well-habituated animals (Morgan, 1973), have repeatedly tested the animals (Einon, Morgan, \& Sahakian, 1975), or have exposed them to long durations of open-field presentation (Sahakian et al., 1977). In the light of these comparisons, it appeared that the conditions and duration of the activity test and the prior experimental history might be especially important in determining the relative activity levels of individually and group-housed

J.C. Dalrymple-Alford was supported by a S.R.C. studentship, Grant 78800404 . The assistance of J. Coe, with the husbandry of the animals, and of P. Wood, with the drawings of the figures, is also gratefully acknowledged. Requests for reprints should be directed to D.B., Department of Psychology, at the above address. rats. It was predicted that, with repeated testing, isolates would be initially less active but later more active than group-housed rats in the open field.

Since there have been reports (Syme, 1973) that different activity measures may not be comparable, two further measures were examined. Nonspecific activity in a novel cage over a 24 -h period was measured, allowing the examination of circadian activity patterns of group-housed and isolated rats; the second measure was rearing (standing up) behavior, which has been proposed as an exploratory measure (Berlyne, 1960) that reflects the level of CNS excitability (Lat, 1965).

Sex differences in the response of differentially housed rats to novelty have received limited investigation. Korn and Moyer (1968) found that individually housed Sprague-Dawley rats of either sex were less active than group-housed counterparts; Archer (1969) reported a similar result only for female Wistar rats, there being no ambulation differences in male differentially housed rats. The influence of sex variables was therefore also examined in this study.

\section{METHOD}

\section{Animals and Housing Conditions}

The animals used were male and female Lister hooded rats (Rattus norvegicus) bred from stock maintained by the Animal Facility, Swansea, Wales. These rats were raised with their mothers in breeding cages (North Kent Plastics; NKP-RB3; $45 \times 28 \times$ $22 \mathrm{~cm}$ ). Differential housing was introduced at weaning (21 days of age). Twenty male and 20 female rats were chosen randomly from different litters and used in two 5-day open-field tests. Half of these animals were housed in single-sex groups of 5 per cage (NKP-RCl; $56 \times 38 \times 18 \mathrm{~cm}$ ); the remainder were placed in standard individual cages (NKP-RM2; $38 \times 23 \times 18 \mathrm{~cm}$ ). A further 10 male and 10 female rats were examined on the activity monitor and in the rearing test (one group-housed female died prior to the rearing test). The housing conditions of these animals were identical to those of the animals tested in the open field, 
except that group-housed animals were reared in NKP-RB3 $(45 \times$ $28 \times 22 \mathrm{~cm}$ ) cages from weaning until 60 days of age, when each group was transferred to a large NKP-RC1 cage. Although the amount of space available per rat is confounded with housing condition, the work of several authors (Einon \& Morgan, 1976; Morley \& Worsham, 1978) suggests that this is not a critical variable. Animals were supplied with food and water ad lib, and were housed in the same room on a 12-h reversed light-dark cycle $(1800$ $0600 \mathrm{~h}$ light) at a temperature of approximately $20^{\circ} \mathrm{C}$. Since the cages were opaqua white polypropylene with flat wire tops, individually housed rats could hear and smell other animals. Once assigned to differential housing conditions, the animals were left undisturbed except for routine maintenance.

\section{Apparatus \\ Open field. The open field measured $75 \times 75 \times 15.5 \mathrm{~cm}$ and was divided into $2515 \times 15 \mathrm{~cm}$ squares. Bright white illumina- tion $(1,700 \mathrm{~lx})$ was produced by three fluorescent tubes beneath a floor of opaque white Perspex. The walls surrounding the field were painted matt black, and the roof of the area was a sheet of perforated zinc; when used in a dark room, this acted as a one-way screen. In addition, white noise of $78 \mathrm{~dB}(\mathrm{~A})$, measured at the cen-} ter, flooded the arena.

Activity monitor. Nonspecific activity of animals was recorded using a "Stoetling" (Chicago, Illinois) activity monitor. Movements of the rat in a cage altered the inductance of magnetic fields produced by coils mounted inside a stage upon which the cage was placed; impulses from this triggered an arbitrary digital printout which was isolated acoustically from the rats.

Rearing test. Rearing behavior was monitored in a cylindrical clear Perspex "rearing chamber," $40.5 \mathrm{~cm}$ high and $20 \mathrm{~cm}$ in diameter; movements of the rat were thus mainly restricted to the vertical direction. These movements altered the ambient capacitance between two circular tin plates, which acted as the floor and ceiling (sensing head to a proximity meter). These plates limited the effective height of the chamber to $30.5 \mathrm{~cm}$. Amplified signals from the proximity meter were recorded on a pen recorder (Washington $400 \mathrm{MD1}$ ) in an adjacent room. The rearing chamber was surrounded by fine white muslin cloth draped over a $50 \times 80 \times 75 \mathrm{~cm}$ metal frame. The only illumination $(26 \mathrm{~lx})$ was produced by four $2.8-\mathrm{W}$ bulbs placed at a height of $20 \mathrm{~cm}$, one at each corner of the rearing chamber's support base. Each bulb was positioned on a wood base behind a piece of black and white, horizontally striped Perspex $(10 \times 12.5 \mathrm{~cm})$.

\section{Procedure}

Open field. The first test began at approximately 63 days of age. Each animal was tested singly for a 4-min trial on 5 consecutive days. These animals were then given a second 5-day test at approximately 100 days of age. Manual records of ambulation (squares traversed with four feet) were taken for consecutive minutes of each trial. At the end of a trial, the rat was returned to the home cage and the floor and walls of the arena were cleansed with a mild disinfectant solution. All testing was conducted between 1000 and $1700 \mathrm{~h}$ and the order of testing was randomized with respect to sex and housing condition.

Activity monitor. Naive animals were monitored individually in a NKP-RM2 $(38 \times 23 \times 13 \mathrm{~cm})$ cage for $24 \mathrm{~h}$ in an otherwise empty, windowless experimental room. A reverse light-dark cycle identical to that in the room containing the rat's home cages $(1800$ $0600 \mathrm{~h}$ lights on) was used. Each rat was placed in a clean cage with fresh sawdust and an ad-lib supply of food and water. The cage was placed on the monitor stage at $1200 \mathrm{~h}$ and was then left undisturbed for the duration of the test. The age of animals at testing varied between 69 and 89 days of age.

Rearing test. Each animal was placed singly in the rearing chamber and monitored for a 40 -min trial. The criterion for scoring a rearing response was taken to be any deflection of the pen corresponding to a movement of the rat to a height of $17.0 \mathrm{~cm}$, for a period of at least $200 \mathrm{msec}$. The chamber was cleaned between tests. The animals used in this test were those animals previously monitored for circadian activity. The age at testing was 140 days.

\section{RESULTS}

\section{Open Field}

A four-way analysis of variance was carried out on the ambulation scores with sex and housing condition as the two main factors and minutes (i.e., within trials) and days (i.e., between trials) as the two repeated measures. When appropriate, significant interactions were analyzed further with regard to their simple main effects (Kirk, 1968). Separate analyses were carried out on the ambulation scores of each 5-day test.

Analysis of the first 5-day test showed that although housing condition had no significant effect $(F<1.0)$, it did interact significantly with both days $[\mathrm{F}(4,144)=$ $4.5, \mathrm{p}<.01]$ and minutes $[\mathrm{F}(3,180)=6.0, \mathrm{p}<.01]$ (see first 5-day test in Figures 1 and 2). Simple main
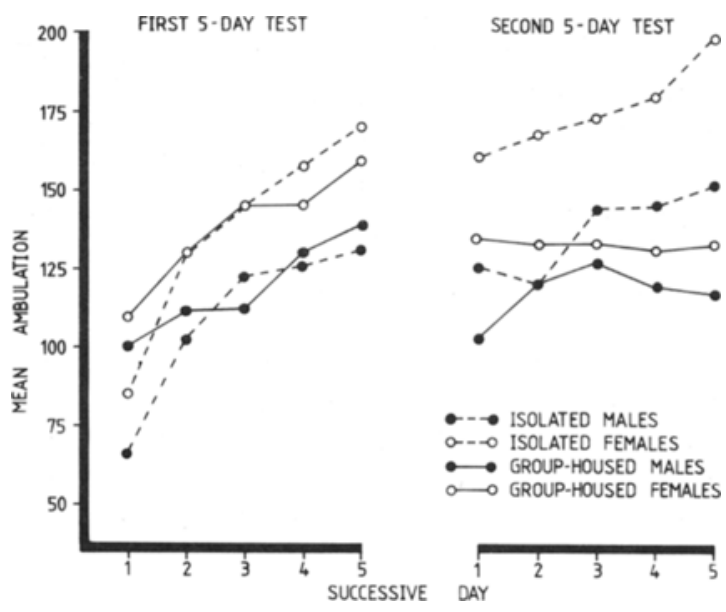

Figure 1. Ambulation for successive days in two 5-day openfield tests by isolated and group-housed male and female rats. Each point is the mean activity of 10 animals in a $4-\mathrm{min}$ period.
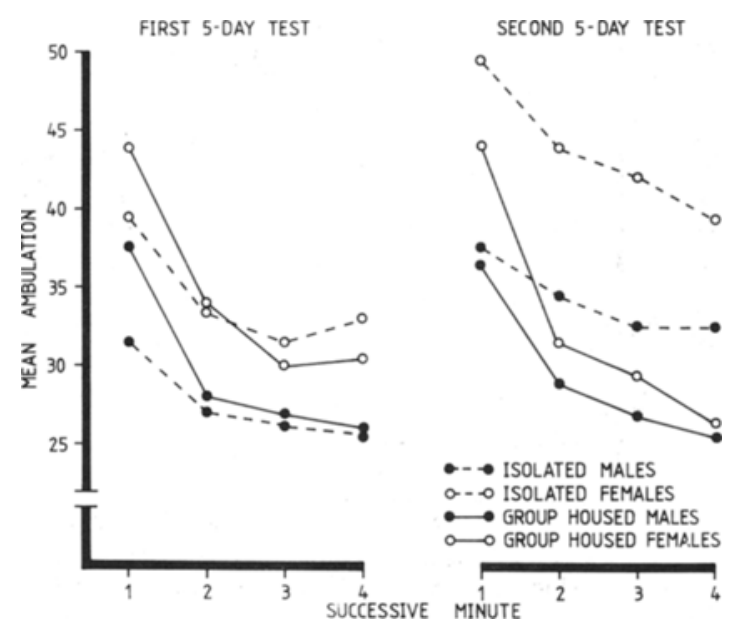

Figure 2. Ambulation for successive minutes in two 5-day openfield tests by isolated and group-housed male and female rats. Each point is the mean activity of 10 animals for each minute of a 4-min daily trial totaled over the 5 days. 
effects analysis of the Housing Condition by Days interaction revealed that individually housed rats crossed fewer squares than did group-housed rats on the first day only $[F(1,180)=11.2, p<.001$, for Day 1 ; $F<1.0$ on the subsequent 4 days]. Both isolated and group-housed rats showed increased ambulation scores over days ( $p<.001$ in both cases). The Housing Condition by Minutes interaction was due to a higher ambulation in group-housed rats in the first minute $[F(1,144)=7.8, p<.01]$; there were no differences between housing conditions in the remaining minutes. It was also found that female rats crossed more squares than did male rats $[F(1,36)=13.1, p<.01]$ but that the sex of animals did not influence any of the findings concerning the two housing conditions.

As expected, the analysis of the second 5-day test revealed that individually housed rats were more active than group-housed rats $[F(1,36)=13.4$, $\mathrm{p}<.01$; Figure 1, second 5-day test]. There was also a significant Day by Housing Condition interaction $[\mathrm{F}(4,144)=2.5, \mathrm{p}<.05]$; specifically, isolated rats showed an increase in ambulation over days $[F(4,144)$ $=5.5, \mathrm{p}<.001]$ but group-housed rats' scores did not change $(F<1.0)$. This second test also showed a Housing Condition by Minute interaction $[F(3,108)$ $=5.1, \mathrm{p}<.01$; Figure 2, second 5-day test]. Grouphoused animals habituated more rapidly within trials. There was no difference between housing conditions after the first minute of testing, but isolates were more active than group-housed animals on each successive minute ( $p<.01$ in all cases). As in the first test, males were less active than females $[F(1,36)=$
9.6, $p<.01]$ and the sex of animals did not interact with housing condition. There was, however, a significant Sex by Minute interaction $[F(3,108)=3.4$, $\mathrm{p}<.05]$. These results are interpreted as being consistent with the idea that individually housed rats are initially less active and later more active than grouphoused animals when tested in the open field.

\section{Activity Monitor}

With this measure, there was heterogeneity of variance across time of day. Since the standard deviations were proportional to the means, raw scores were transformed to $\log _{10}$ prior to analysis of variance (Kirk, 1968, p. 65). As expected, animals exhibited a clear circadian activity $[\mathrm{F}(23,368)=28.1, \mathrm{p}<.01]$. More notably, individually housed rats showed significantly higher levels of nonspecific activity than group-housed rats $[F(1,16)=15.0, p<.01]$, and the effect of housing condition interacted with time of day $[F(23,368)=2.4, p<.01$; Figure 3$]$. Analysis of the simple main effect of housing condition at each successive hour revealed isolates to be significantly more active than group-housed rats at 1500,1600, 1900 (the period during which lights came on), 2300, 0100 , and $0200 \mathrm{~h}(\mathrm{p}<.05$ in all cases). Group-housed rats were more active than isolates during one hour only $(2100 \mathrm{~h}, \mathrm{p}<.01)$. Individually housed rats thus habituated to the novel environment more slowly than group-housed rats. Also, group-housed rats were less active than isolates during some of the "lights on" period. The sex of the animals did not influence any of these findings.

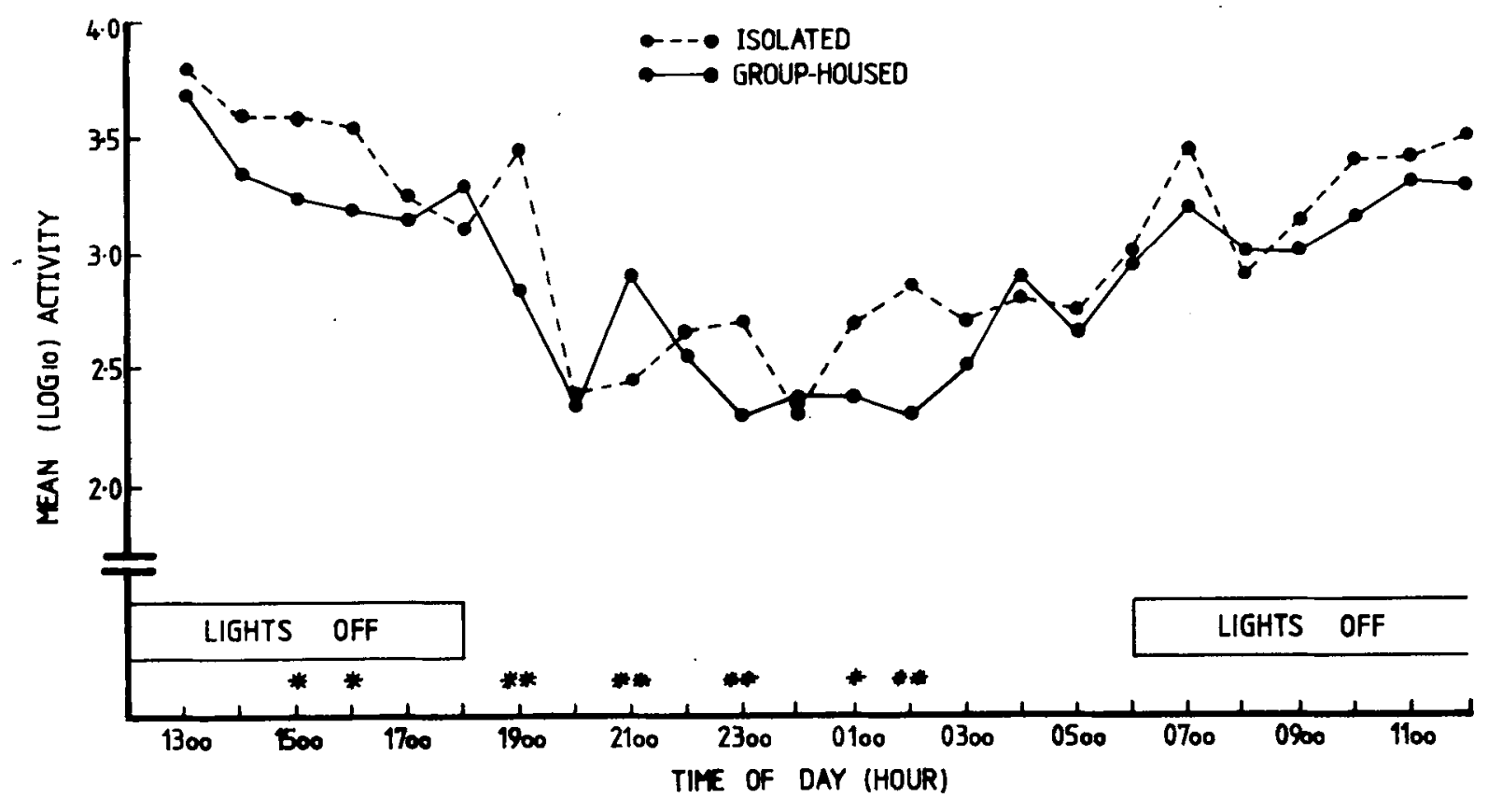

Figure 3. Nonspecific cage activity in isolated and group-housed rats over $24 \mathrm{~h}$. Each point is the mean of 1-h activity for 10 animals, 5 male and 5 female. ${ }^{\star}=p<.05,{ }^{\star \star}=p<.01$. 


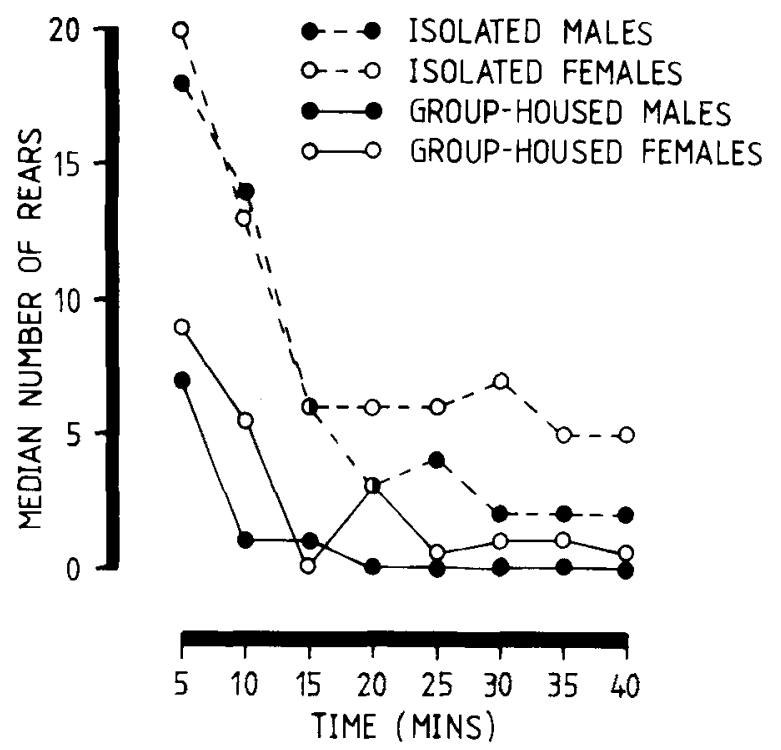

Figure 4. Number of rears made in a "rearing chamber" by isolated and group-housed male and female rats. Each point represents the median number of rears made in consecutive 5-min periods of a single 40 -min trial.

\section{Rearing Behavior}

Group-housed rats exhibited little or no rearing after $10-15 \mathrm{~min}$, whereas isolated rats continued to rear throughout the 40-min trial (Figure 4). The number of rears totaled over the 40 -min period were analyzed by Mann-Whitney $U$ tests (two-tailed). Interactions between housing categories on these scores were additionally analyzed over consecutive 5-min intervals using the nonparametric methods described by Still (1967).

As expected, isolated rats reliably made more rears than group-housed rats, irrespective of sex, over the 40-min trial (males, $\mathrm{U}=.5, \mathrm{p}<.01$; females, $\mathrm{U}=$ $0, \mathrm{p}<.01)$. There was a significant interaction between housing condition and time in both sexes (males, $U=1, p<.01$; females, $U=2, p<.05$ ); this Housing Condition by Time interaction reflects the rapid decline of relatively low rearing levels in grouphoused rats. Also, isolated females made more rears than isolated males $(U=4, p<.05)$, but there was no Sex by Time Interval interaction in the isolated animals. There was no sex difference, or Sex by Time Interval interaction in the rearing behavior of grouphoused rats.

\section{DISCUSSION}

The demonstration that early open-field ambulation of naive individually housed rats is lower than that of naive group-housed rats is consistent with several studies (see the introduction). This finding has often been interpreted in terms of a greater "emotional reactivity" of isolates (Ader \& Friedman, 1964; Stern et al., 1960; Taylor, 1969), although a more precise suggestion (Archer, 1970) is that naive isolated rats exhibit reduced exploratory behavior compared with group-housed animals due to higher levels of "fear responses," such as freezing. No direct measures of freezing behavior were taken in this study, but isolates in particular showed considerable immobility at the beginning of open-field testing. This study clearly shows that the relatively lower activity of isolates is transitory in nature; it did not survive Day 1 of the first open-field test and was not reintroduced by a 5 week period before retesting.

There is evidence that housing rats individually produces at least two separate effects. Einon and Morgan (1977) have reported a "critical period" (25-45 days of age) for isolation-induced open-field hyperactivity, which, they claim, can be dissociated from the increased emergence times of isolates. Only the latter effect was produced by isolation at any age and reversed by subsequent group housing. Because the longer emergence times of isolates may be due to "fear responses" rather than to a slower habituation to the emergence chamber as suggested previously (Brain \& Benton, 1979; Morgan, Einon, \& Nicholas, 1975), it is possible that isolation in rats affects the processes regulating "fear responses" in addition to those influencing exploratory behavior. The decline (through repeated testing, handling, etc.) in initial fear responses reveals a characteristic and more permanent hyperactivity in individually housed rats, reflected in the high ambulation scores of isolated relative to group-housed rats in the second open-field test.

It follows, from the above discussion, that when the test conditions are insufficient to elicit fear responses, isolates are more exploratory than grouphoused animals, an assertion supported by the highactivity monitor behavior and elevated rearing scores of isolated compared with group-housed rats (Figures 3 and 4). The broad interpretation that individual housing produces more exploratory behavior than does group-housing is not without its problems. The present findings indicate that exploration of a novel environment is enhanced by individual housing. This finding does not necessarily mean, however, that isolation has a similar effect on exploration directed towards discrete novel stimuli ("inspective exploration," Berlyne, 1960). The hyperactivity of isolates is associated with the former type of exploration but is found to interfere with the latter type (Sahakian et al., 1977). It is therefore not surprising that isolates are inferior to group-housed animals on measures of manipulatory, but not nonmanipulatory, contacts with objects (Einon \& Morgan, 1976) and that isolates spend less time investigating objects in a hole-board (File, 1978).

The present finding also provides much support for the claim (Einon \& Morgan, 1976; Einon et al., 
1975) that the hyperactivity of isolates reflects their poorer ability to adapt and habituate to novelty. Those processes energizing an increase in ambulation over days in the first open-field test apparently still influenced isolated but not group-housed rats in the second 5-day test. Group-housed rats habituated more rapidly than isolated rats over minutes in both open-field tests. Although this effect in the first 5-day test may have reflected a suppressed ambulation of isolates in the first minute, habituation differences were clearly observed in the second 5-day test (Figure 2). Both isolated and group-housed rats habituated rapidly in terms of rearing behavior, but, in contrast with grouphoused animals, rearing in isolates was more persistent and was not suppressed over the lengthy test period as completely as it was in group-housed animals.

Consistent with the report that isolates spend less time asleep (Tagney, 1973), the nonspecific activity measure showed that isolates were more active over $24 \mathrm{~h}$ than their group-housed counterparts (Figure 3). However, the activity differences during the first "lights off" period (1300-1800 h) can be interpreted as further evidence of retarded habituation in isolated animals; isolates did not significantly differ from group-housed animals during any part of the second "lights off" period (0700-1200 h). Both housing categories were relatively inactive during "lights on" (1900-0600 h), but isolated animals tended to be reliably more active than group-housed animals during some of this period. The only previous investigation of circadian differences of group-housed and isolated rats reported a similar result: isolates consumed more food than group-housed rats during the day, but not during the night (Morgan \& Einon, 1975). Whether these differences in diurnal variation influence the nature of any other isolation-induced changes remains to be determined, as does the possibility that other diurnal variations, for example, adrenocortical activity, are affected by differential housing.

There was no evidence that the sex of animals influenced the activity differences of housing categories in the open field and in a novel cage. However, there was an indication that individual housing affected female more than male rearing behavior.

This study confirms that housing rats in individual cages has pronounced effects on their activity levels. In general, isolates were found to be consistently hyperactive compared with group-housed rats in three different situations, each using a different measure. In addition, the apparent inconsistencies arising from comparisons of previous open-field studies have also been clarified: in a stressful situation, naive isolates are less active only at the beginning of testing; they become relatively more active than group-housed animals later.

\section{REFERENCES}

ADER, R. Effects of early experience and differential housing on behavior and susceptibility to gastric erosions in the rat. Journal of Comparative and Physiological Psychology, 1965, 60, 233-238.

Ader, R., \& Friedman, S. B. Social factors affecting emotionality and resistance to disease in animals: IV. Differential housing, emotionality, and Walker 256 carcinosarcoma in the rat. Psychological Reports, 1964, 15, 535-541.

Archer, J. Contrasting effects of group housing and isolation on subsequent open field exploration in laboratory rats. Psychonomic Science, 1969, 14, 234-235.

Archer, J. Effects of population density on behaviour in rodents. In J. H. Crook (Ed.), Social behaviour in birds and mammals. Essays on the social ethology of animals and man. London: Academic Press, 1970.

Berlyne, D. E. Conflict, arousal and curiosity. New York: McGraw-Hill, 1960.

Brain, P., \& Benton, D. The interpretation of physiological correlates of differential housing in laboratory rats. Life Sciences, 1979, 24, 99-116.

Domjan, M., Schorr, R., \& Best, M. Early environmental influences on conditioned and unconditioned ingestinal and locomotor behaviors. Developmental Psychobiology, 1977, 10, 499-506.

Einon, D. F., \& Morgan, M. J. Habituation of object contact in socially-reared and isolated rats (Rattus norvegicus). Animal Behaviour, 1976, 24, 415-420.

Einon, D. F., \& Morgan, M. J. A critical period for social isolation in the rat. Developmental Psychobiology, 1977, 10, 123-132.

Einon, D. F., Morgan, M. J., \& Sahakian, B. J. The development of intersession habituation and emergence in sociallyreared and isolated rats. Developmental Psychobiology, 1975, 8, 553-560.

File, S. E. Exploration, distraction, and habituation in rats reared in isolation. Developmental Psychobiology, 1978, 11, 73-81.

Kır., R. E. Experimental design: Procedures for the behavioral sciences. Belmont, Calif: Wadsworth, 1968.

Korn, H. J, \& MoYer, K. E. Behavioral effects of isolation in the rat: The role of sex and time of isolation. Journal of Genetic Psychology, 1968, 113, 263-273.

LAT, J. The spontaneous exploratory reactions as a tool for psychopharmacological studies. A contribution towards a theory of contradictory results in psychopharmacology. In $\mathrm{M}$. Y. Mikhelison \& V. G. Longe (Eds.), Pharmacology of conditioning, learning and retentions. New York: Pergamon Press, 1965.

Morgan, M. J. Effects of post-weaning environment on learning in the rat. Animal Behaviour, 1973, 21, 429-442.

Morgan, M. J., \& Einon, D. F. Incentive motivation and behavioral inhibition in socially-isolated rats. Physiology \& Behavior, 1975, 15, 405-409.

Morgan, M. J., Einon, D. F., \& Nicholas, D. The effects of isolation rearing on behavioral inhibition in the rat. Quarterly Journal of Experimental Psychology, 1975, 27, 615-634.

Morley, B. J., \& Worsham, E. The effects of prolonged handling, scopolamine, and physostigmine on the activity of isolated and socially reared rats. Physiological Psychology, 1978, 6, 83-88.

MOYER, K. E., \& KoRN, J. H. Behavioral effects of isolation in the rat. Psychonomic Science, 1965, 3, 503-504.

Nishikawa, T., \& Tanaka, M. Altered behavioral responses to intense footshock in socially isolated rats. Pharmacology, Biochemistry \& Behavior, 1978, 8, 61-67.

Sahakian, B. J., Robbins, T. W., \& Iversen, S. D. The effects 
of isolation rearing on exploration in the rat. Animal Learning \& Behavior, 1977, 5, 193-198.

Speiser, Z., \& Weinstock, M. The influence of propanol on abnormal behaviour induced in rats by prolonged isolation: An animal model for mania? British Journal of Pharmacology, 1973, 48, 348-349.

STILL, A. W. Use of orthogonal polynomials with nonparametric tests. Psychological Bulletin, 1967, 68, 327-329.

Stern, J. A., Winokur, G., Eisenstein, A., Taylor, R., \& SLY, M. The effect of group vs individual housing on behaviour and physiological responses to stress in the albino rat. Journal of Psychosomatic Research, 1960, 4, 185-190.
Syme, L. A. Social isolation at weaning: Some effects on two measures of activity. Animal Learning \& Behavior, 1973, 1, 161-163.

TAGNEY, J. Sleep patterns related to rearing rats in enriched and impoverished environments. Brain Research, 1973, 53, 353-361.

TAYLOR, J. The effects of population density upon correlates of emotionality and learning efficiency. Journal of General Psychology, 1969, 80, 205-218.

(Received for publication January 28, 1980; revision accepted July 9,1980 .) 